\title{
Numerical investigation of evaporation phenomena of liquid fuel jet atomization in crossflow
}

\author{
Kenya Kitada*, Jian Wen, Ryoichi Kurose \\ Department of Mechanical Engineering and Science, Kyoto University, Kyoto, Japan \\ ${ }^{*}$ Corresponding author email : kitada.kenya.87c@st.kyoto-u.ac.jp
}

\begin{abstract}
The liquid jet atomization and evaporation process in a crossflow is numerically investigated using an Eulerian-Lagrangian framework. The crossflow is set as an oscillating condition, which is generally found occurring in the practical aircraft engines due to combustion instability, to clarify the oscillation effects on the atomization and evaporation characteristics for a fixed liquid jet velocity. Continuous variations of atomization characteristics under the multimode breakup are observed when the crossflow velocity keeps varying. A detailed investigation on liquid jet trajectory and its relation to atomization morphology is presented. Moreover, the oscillating crossflow shows better atomization and evaporation properties upon comparing the droplet profiles such as the Sauter mean diameter and the arithmetic diameter, as well as the fuel vapor distribution.
\end{abstract}

\section{Keywords}

Atomization; Evaporation; Oscillating crossflow; Eulerian-Lagrangian framework

\section{Introduction}

Spray combustion is widely utilized in different energy generators such as rocket engines, aircraft engines as well as diesel engines. Compared to the free jet injected into quiescent surrounding air such as conventional diesel engines, the transverse injection of the liquid fuel jet into crossflow has received more focus due to its practical application in the air-breathing propulsion systems, such as ramjet combustor and gas turbine combustors for larger energy density.

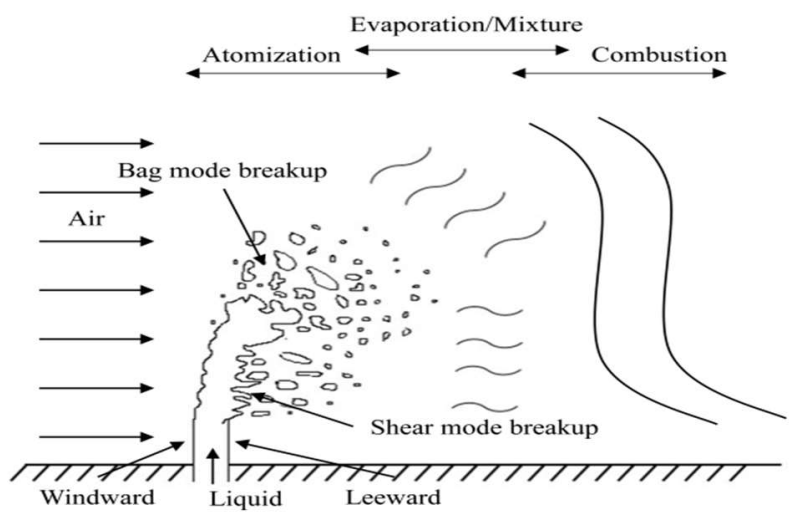

Figure 1. Schematic of spray combustion in crossflow.

Figure 1 shows the schematic of spray combustion in crossflow, indicating that the liquid jet would breakup into ligaments and small droplets during the atomization process, and then the generated droplets keep dispersing and evaporating by the carrying hot air. The vaporized fuel vapor is further mixed with the surrounding air at downstream region and finally the combustion takes place to supply power for the engines [1]. 
Focusing on the process of atomization, since the fuel jet is perpendicularly injected into the crossflow, it would be deflected along with the air stream and the generation of some wrinkles or disturbances will occur at the jet column surface. Subsequently, these disturbances are accelerated by the crossflow and develop into large waves. Finally, these waves are too large to sustain themselves and breakup into ligaments and droplets. This mechanism is referred to as the bag mode breakup [2]. When the air velocity is further increased thus leading to the increased air shearing forces, some wave instabilities would also occur on the jet column lateral surface at the vicinity of the nozzle. While the high velocity air passes through the jet column, the ligaments and smaller droplets are stripped out of the column from the shear forces. This is referred to as the shear mode breakup [2].

Bag mode breakup and shear mode breakup could take place simultaneously that could be regarded as a competitive mechanism, both of which result from aerodynamic forces. Therefore, for the primary breakup, it is the same situation as the secondary breakup of the droplet. The aerodynamic Weber number $\left(W e_{g}=\rho_{g} v_{g}{ }^{2} d_{0} / \sigma\right)$ and liquid to gas momentum flux ratio $\left(M=\rho_{I} v_{l}^{2} / \rho_{g} v_{g}{ }^{2}\right)$, are found to have significant influence on jet development [2-4], where $\rho$ is the density, $V$ the velocity, $d_{0}$ the nozzle diameter, $\sigma$ the surface tension, and subscript $g$ and $/$ refer to gaseous crossflow and liquid jet physical properties, respectively. Many different experiments and numerical researches [1-6] share the common understanding that the breakup characteristics of liquid jet in crossflow is dominated by the aerodynamic Weber number and liquid to gas momentum flux ratio. Whereas, fluid viscosity, liquid-air density ratio as well as temperature have minor effects on the liquid jet evolution regardless of cavitation or turbulence.

When combustion instability occurs during the combustion process, the induced thermosacoustics could also influence the fuel atomization process, by producing either a positive or negative feedback on it, thereby resulting in better or worse atomization characteristics [7]. However, all the understandings of atomization process mentioned above are obtained by conducting the experiments or numerical investigations almost under the steady crossflow condition by adjusting $W e_{g}$ and $M$ over a large range. Even though some of them have tried modulated crossflow velocity profiles under different frequencies, their researches $[8,9]$ have shown totally different results on liquid jet trajectory and droplet size distribution So, the underlying mechanism of liquid jet atomization under oscillating crossflow velocity still remains a mystery. It is still hard to judge which kind of atomization would be better for the final combustion.

The purpose of this study is to investigate the atomization-evaporation process of liquid fuel jet spray in the oscillating crossflow under the frequency commonly found in practical gas turbine combustors by using an Eulerian/Lagrangian framework, in which both continuous gas and liquid phases are solved in an Eulerian manner, and the generated dispersed droplets are tracked in a Lagrangian manner to consider the effects of evaporation. Therefore, effects of oscillating condition could be directly understood by comparing the droplet size distribution and fuel vapor profiles.

\section{Numerical Methods and Computational Setup}

In present study, both liquid and gas phases are treated as incompressible fluids and solved in the Eulerian manner by using in-house code, $\mathrm{FK}^{3}[1,10]$. The governing equations of mass and momentum are utilized for continuous liquid and gas phases for atomization, and the governing equations of enthalpy as well as chemical species are only implemented for gas phase to investigate the transport of air and fuel vapor mixture after droplets' evaporation. 


$$
\begin{aligned}
& \rho \nabla \cdot \boldsymbol{u}=S_{\rho}, \\
& \rho\left(\frac{\partial \boldsymbol{u}}{\partial t}+\boldsymbol{u} \cdot \nabla \boldsymbol{u}\right)=-\nabla P+\nabla \cdot(2 \mu \boldsymbol{S})+\boldsymbol{F}_{\sigma}+S_{\rho \boldsymbol{u}}, \\
& \rho\left(\frac{\partial h}{\partial t}+\boldsymbol{u} \cdot \nabla h\right)=\nabla \cdot\left(\frac{\lambda}{c_{p}} \nabla h\right)+S_{\rho h}, \\
& \rho\left(\frac{\partial Y_{k}}{\partial t}+\boldsymbol{u} \cdot \nabla Y_{k}\right)=\nabla \cdot\left(D_{k} \nabla Y_{k}\right)+S_{\rho Y_{k}},
\end{aligned}
$$

Here, $\rho$ is the density, $\boldsymbol{u}$ the velocity vector, $P$ the pressure, $\mu$ the viscosity, $\boldsymbol{S}$ the rate-ofstrain tensor $\boldsymbol{S}_{i j} \equiv\left(\partial_{i} u_{j}+\partial_{j} u_{i}\right) / 2, \boldsymbol{F}_{\sigma}$ the source term of surface tension, $h$ the specific enthalpy, $\lambda$ the thermal conductivity, $C_{p}$ the specific heat capacity at constant pressure, $Y_{k}$ and $D_{k}$ are the mass fraction and the mass diffusion coefficient of the kth species, respectively. $S_{\rho}$, $S_{\rho u}, S_{\rho h}$, and $S_{\rho Y_{k}}$ are the source terms of mass, momentum, energy, and chemical species for the interaction between gas phase and fuel droplets calculated by the following equations.

$$
\begin{aligned}
& S_{\rho}=-\frac{1}{\Delta V} \sum_{N} \frac{d m_{d}}{d t}, \\
& S_{\rho \boldsymbol{u}}=-\frac{1}{\Delta V} \sum_{N} \frac{d m_{d} \boldsymbol{u}_{d}}{d t}, \\
& S_{\rho h}=-\frac{1}{\Delta V} \sum_{N} \frac{d m_{d} h_{d}}{d t}, \\
& S_{\rho Y_{k}}= \begin{cases}-\frac{1}{\Delta V} \sum_{N} \frac{d m_{d}}{d t} & \text { for fuel } \\
0 & \text { for other }\end{cases}
\end{aligned}
$$

Here, $\Delta V$ is the volume of the unit grid, $\mathrm{N}$ the number of droplets in the grid, and $m_{d}, \boldsymbol{u}_{d}$, and $h_{d}$ are the mass, velocity, and specific enthalpy of the droplet, respectively.

The fuel droplets generated by atomization are then tracked in a Lagrangian manner so that the droplets' profiles such as position, $x_{d}$, velocity, $\boldsymbol{u}_{d}$, temperature, $T_{d}$, as well as mass, $m_{d}$ are solved by the following governing equations.

$$
\begin{aligned}
\frac{d x_{d}}{d t} & =\boldsymbol{u}_{d}, \\
\frac{d \boldsymbol{u}_{d}}{d t} & =\frac{f_{1}}{\tau_{d}}\left(\boldsymbol{u}-\boldsymbol{u}_{d}\right), \\
\frac{d T_{d}}{d t} & =\frac{N u}{3 P r}\left(\frac{C_{p}}{c_{p, d}}\right)\left(\frac{f_{2}}{\tau_{d}}\right)\left(T-T_{d}\right)+\frac{1}{m_{d}} \frac{d m_{d}}{d t} \frac{L_{v}}{C_{p, d}}, \\
\frac{d m_{d}}{d t} & =-\frac{S h}{3 S c} \frac{m_{d}}{\tau_{d}} \ln \left(1+B_{M}\right),
\end{aligned}
$$

Here, $f_{1}$ and $f_{2}$ are the correction coefficients for Stokes drag and heat transfer, $\tau_{d}$ the particle response time, $T$ the gas temperature, $T_{d}$ the droplet temperature, $L_{v}$ the latent heat of evaporation of the droplet at temperature $T_{d}, C_{p}$ and $C_{p, d}$ the specific heat of gas and fuel droplet, $\mathrm{Nu}$ the Nusselt number, $\mathrm{Pr}$ the Prandtl number, $S h$ the Sherwood number, $S c$ the Schmidt number, and $B_{M}$ the mass transfer number. The detailed information of these parameters could be found in our previous works and the non-equilibrium Langmuir-Knudsen evaporation model utilized for the droplet evaporation has been validated in Kitano's works $[11,12]$.

A coupled level-set and volume-of-fluid (CLSVOF) method [13] is implemented to capture the interface between gas and liquid in the Eulerian manner. The weighted line interface calculation (WLIC) scheme [14] is used for VOF shape reconstruction and level-set method is coupled to smooth the interface at each step to acquire accurate surface tension. The surface tension is then calculated by the continuum surface force (CSF) model [15]. The transport 
equation of VOF method, the reinitialization process of level-set method as well as the solution of CSF model could be also found in our previous work [1].

The CLSVOF method is only implemented during the primary breakup in the atomization process and droplets as well as ligaments are then generated to disperse in the flow field. In this study, the Eulerian/Lagrangian transformation is triggered at each step to transform Eulerian components to Lagrangian droplets precisely for the evaporation investigation. A tagging method is generally referred to Herrmann's work [16], and the judgement to properly transform spherical Eulerian components instead of ligaments to Lagrangian particles could be also found in Li's work [17].

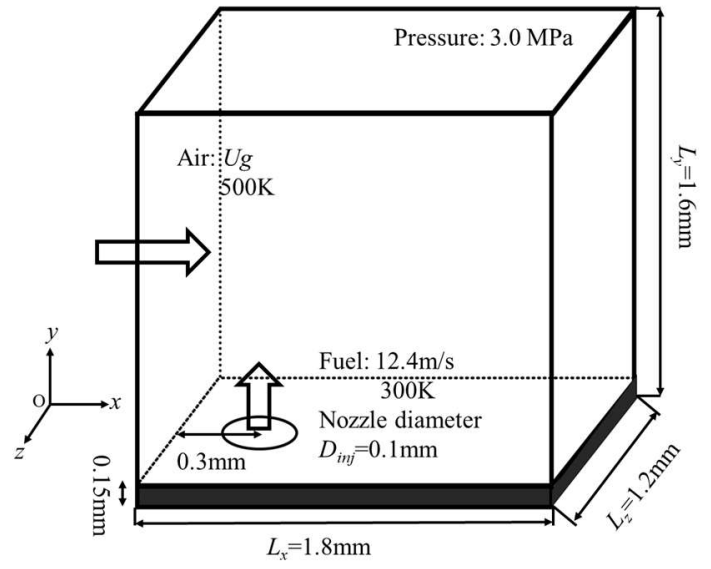

(a)

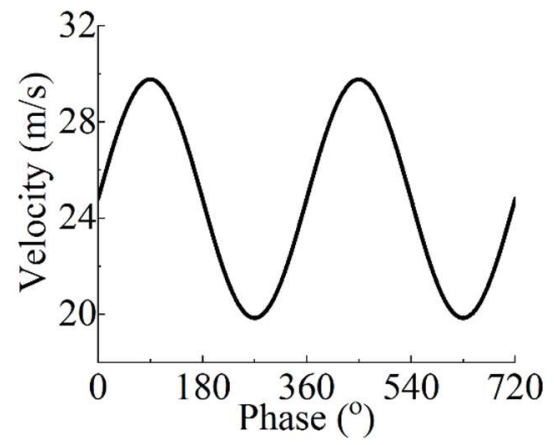

(b)

Figure 2. Schematic of computational domain and inlet velocity profile of oscillation crossflow.

Figure 2(a) shows the schematic of computational domain of present study. The liquid jet is injected into the domain from bottom side where a non-slip boundary wall with a height of $0.15 \mathrm{~mm}$ is set for the liquid flow evolution. The jet nozzle has a diameter $D_{i n j}$ of $0.1 \mathrm{~mm}$, which is located at $0.3 \mathrm{~mm}$ downstream of the air stream inlet. The whole computational domain has a volume of $1.8 \mathrm{~mm} \times 1.6 \mathrm{~mm} \times 1.2 \mathrm{~mm}$ which contains $360 \times 320 \times 240$ grid points in $\mathrm{x}, \mathrm{y}$, and $\mathrm{z}$ direction, respectively. The total grid points in the computational domain are 27.6 million, of which the uniform mesh size of $5 \mu \mathrm{m}$ is employed. The mesh independence validation has been checked in our previous work [1] by adopting a refined uniform mesh size of $2.5 \mu \mathrm{m}$ so that the gas phase is solved in a direction numerical simulation (DNS) and the interface between gas and liquid phases could be regarded as well-resolved.

In order to evaluate the atomization process stimulated by the oscillating crossflow, the evaporation effects are considered in this study by comparing the droplet profiles and fuel vapor distribution. Therefore, the hot air of $500 \mathrm{~K}$ and cold liquid of $300 \mathrm{~K}$ are utilized in the high-pressure condition of $3.0 \mathrm{MPa}$ which is close to the practical condition. Kerosene is used as the jet fuel which is injected into the domain using a parabolic velocity profile with the mean value of $12.4 \mathrm{~m} / \mathrm{s}$. Air is used as the crossflow using a uniform velocity profile of $24.8 \mathrm{~m} / \mathrm{s}$ with the oscillation frequency of $500 \mathrm{~Hz}$. The amplitude of fluctuating air velocity is $20 \%$ of the average velocity and the oscillating velocity profile is made to fit with a sine curve as shown in Figure 2(b). The physical properties and dominant parameters for the liquid jet in crossflow configuration are listed in Table 1. Kerosene is assumed to be an equivalent single species $\left(\mathrm{C}_{9.7396} \mathrm{H}_{20.0542}\right)$ of $\mathrm{C}_{10} \mathrm{H}_{22}(76.7 \mathrm{wt} \%), \mathrm{C}_{9} \mathrm{H}_{12}(13.2 \mathrm{wt} \%)$ and $\mathrm{C}_{9} \mathrm{H}_{18}(10.1 \mathrm{wt} \%)$. In addition, the aerodynamic Weber number ranges from 29.44 to 66.24 , which could be regarded as multimode breakup where shear mode breakup and bag mode breakup would occur 
simultaneously. To capture the secondary breakup, the Taylor Analysis Breakup (TAB) model was applied, but little effects had been observed because the small computational domain could not afford enough time to trigger the secondary breakup, and hence the TAB model is neglected in this study.

The computational cost for the oscillating case, performed with 1200 cores on the Kyoto University Supercomputer (Cray XC40) is 1.4 Million CPU hours (real time 1170h).

Table 1 - Detailed parameters for the oscillating crossflow simulation.

\begin{tabular}{|l|l|}
\hline \multicolumn{2}{|c|}{ Physical properties } \\
\hline Liquid fuel & Kerosene \\
\hline Crossflow gas & Air \\
\hline Liquid nozzle diameter, $D_{\text {inj }}(\mathrm{mm})$ & 0.1 \\
\hline Liquid jet velocity, $u_{l}(\mathrm{~m} / \mathrm{s})$ & 12.4 \\
\hline Liquid jet temperature, $T_{l}(\mathrm{~K})$ & 300 \\
\hline Liquid jet viscosity, $\mu_{l}(\mathrm{~Pa} \cdot \mathrm{s})$ & $2.87 \times 10^{-3}$ \\
\hline Liquid jet density, $\rho_{l}\left(\mathrm{~kg} / \mathrm{m}^{3}\right)$ & 848 \\
\hline Crossflow velocity, $u_{g}(\mathrm{~m} / \mathrm{s})$ & $19.84-29.76$ \\
\hline Crossflow temperature, $T_{g}(\mathrm{~K})$ & 500 \\
\hline Crossflow viscosity, $\mu_{g}(\mathrm{~Pa} \cdot \mathrm{s})$ & $2.72 \times 10^{-5}$ \\
\hline Crossflow density, $\rho_{g}\left(\mathrm{~kg} / \mathrm{m}^{3}\right)$ & 20.8 \\
\hline Crossflow frequency, $F_{g}(\mathrm{~Hz})$ & 500 \\
\hline Liquid-gas surface tension, $\sigma(\mathrm{N} / \mathrm{m})$ & 0.03 \\
\hline Ambient pressure, $P(\mathrm{MPa})$ & 3.0 \\
\hline Aerodynamic Weber number, $W e_{g}$ & $29.44-66.24$ \\
\hline Jet Ohnesorge number, Oh & 0.06 \\
\hline Liquid-gas momentum flux ratio, $M$ & $7.08-15.93$ \\
\hline
\end{tabular}

\section{Results and Discussion}

Figure 3 shows the three dimensional visualization figures of instantaneous iso-surface of the levlelset value equal to 0 , which represents the interface between liquid and gas phases. The comparison is given here for the different phases of $90^{\circ}$ and $270^{\circ}$, when the crossflow velocity reaches the lowest and highest values, respectively. It is clear that the liquid jet trajectory is dominated by the instantaneous crossflow velocity which decides the aerodynamic Weber number and liquid-gas momentum flux ratio. When the crossflow velocity reaches its lowest value, that is at the phase of $270^{\circ}$, the aerodynamic force is weak due to which the deflection by air stream is smaller and liquid jet could penetrate higher. In this case, fewer ligaments are observed to be stripped out of the liquid column, so the liquid column could sustain its shape even when it reaches downstream. However, when crossflow velocity increases, the aerodynamic force is enhanced and hence more ligaments could be observed. An obvious change is that the bifurcations near the fuel nozzle, which display moderate elongated morphology at the phase of $270^{\circ}$, are almost diminished at the phase of $90^{\circ}$, indicating extremely violent atomization characteristics near the fuel nozzle. Due to the great mass loss at the upstream region, the liquid jet exchanges larger momentum with the crossflow compared to the case in $270^{\circ}$, which makes it difficult to keep its shape in the downstream location, and then the liquid column tends to break down into several discountineous components. Also, at the phase of $270^{\circ}$, more larger Eulerian droplets, which are the white components away from the liquid jet, could be observed. These larger droplets are still too 
large to be transformed into Lagrangian droplets, and would then breakup into smaller droplets. Therefore, more red Lagrangian droplets with big size could be found around these larger droplets. Whereas, at the phase of $90^{\circ}$, more smaller droplets are generated. Due to increased aerodynamic Weber number at the phase of $90^{\circ}$, shear mode breakup becomes dominating in the crossflow atomization and then droplets tend to be stripped out of liquid column instead of bag-like breakup in bag mode breakup. Each red circle depicted on the liquid jet is an aritificial region called a transition point in our previous study, beyond which the breakup characteristics could be found to transit from shear mode breakup to bag mode breakup.

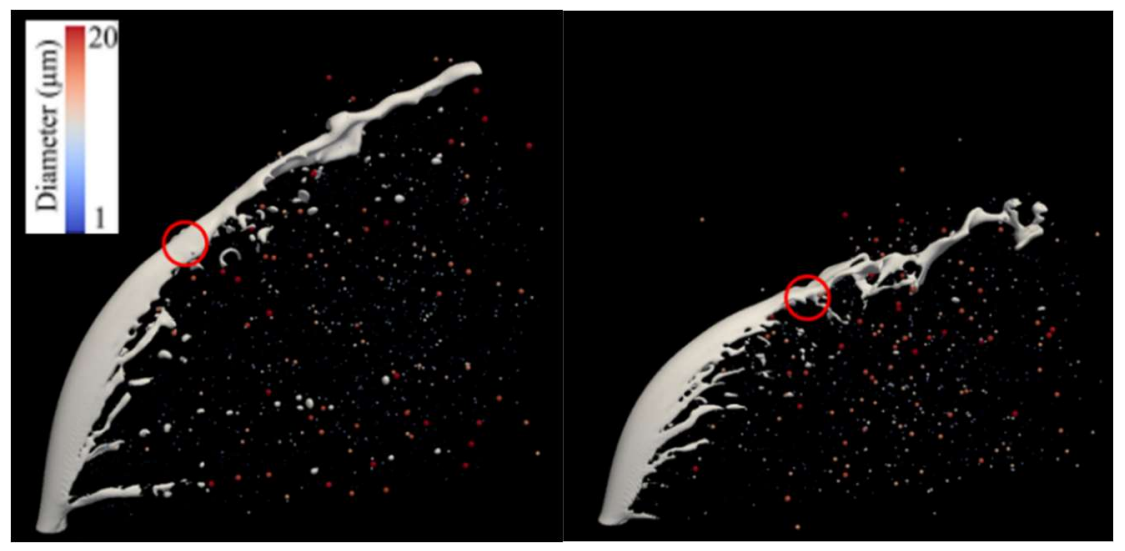

(a) $270^{\circ}$

(b) $90^{\circ}$

Figure 3. Three dimensional snapshots of instantaneous iso-surface of level-set value $\varphi=0$ at different phases of (a) $270^{\circ}$ and (b) $90^{\circ}$. Particle colour indicates the droplet size.

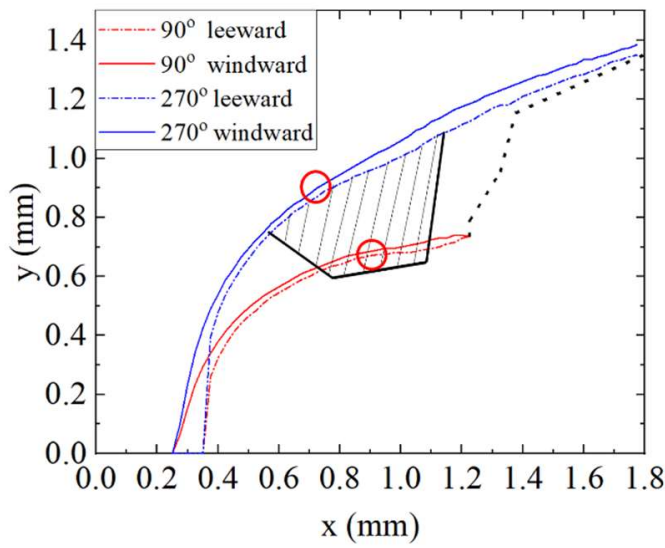

Figure 4. Liquid jet trajectories on the central $x-y$ plane $(z=0.0 \mathrm{~mm})$.

Figure 4 shows the time-averaged liquid jet trajectory on the central $x-y$ plane $(z=0.0 \mathrm{~mm})$. The depicted trajctories represent the liquid jet with the VOF value larger than 0.5 so that the trajectories could be evaluated as the penetrations during different phases. The black dotted line in the figure depicts the farthest points of the time-averaged liquid jet trajectories at the different phases. Except for the phase of $270^{\circ}$, the liquid jet reaches its end in the computational domain and both breakup modes are observed in the simulation. In addition, the shaded region in Figure 4 represents the region where the probability of VOF value to be less than 0.5 is high. It is the region where the discontinuous Eulerian components could be observed and stronger atomization process is likely to occur. It could be found that the 
transition point where the atomization chatacteristics transit from shear mode breakup to bag mode breakup exactly locates in this region.

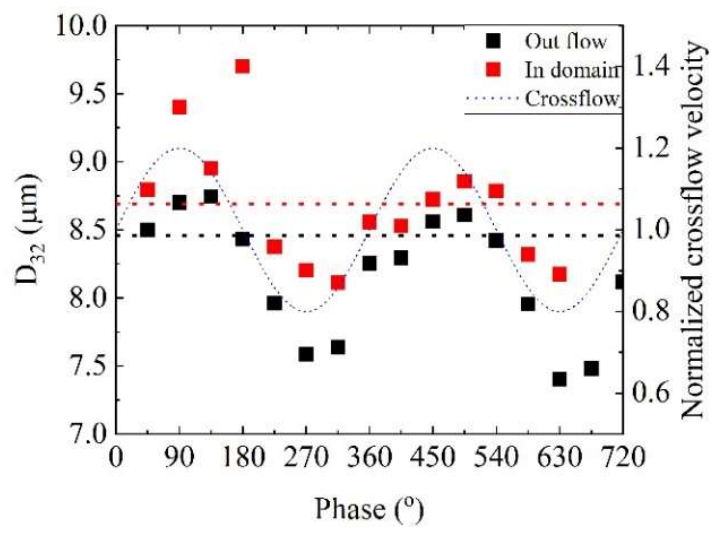

(a) $\mathrm{D}_{32}$

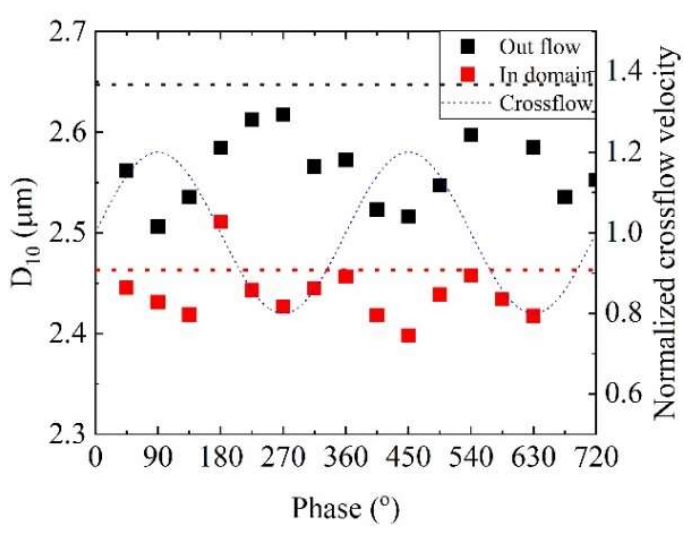

(b) $\mathrm{D}_{10}$

Figure 5. Fluctuations of droplet sizes $\left(D_{32}\right.$ and $\left.D_{10}\right)$ in the domain $(0.0 \mathrm{~mm}<x<1.8 \mathrm{~mm})$ and downstream region $(1.5 \mathrm{~mm}<x<1.8 \mathrm{~mm})$ of the oscillating and steady cases. Blue dotted line indicates crossflow oscillation for comparison of phase delay.

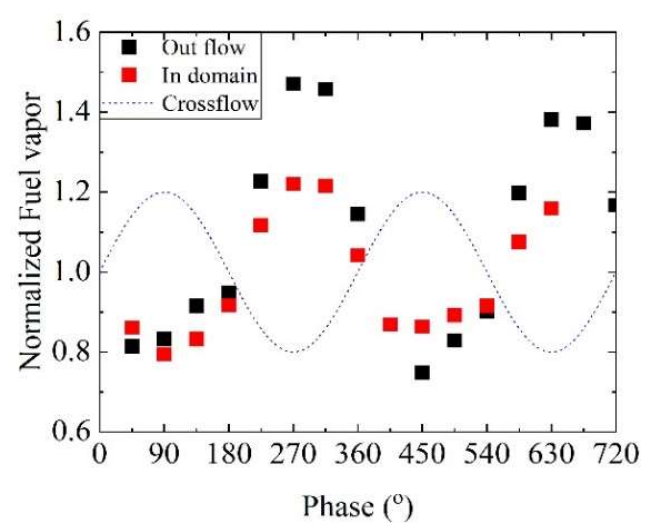

Figure 6. Fluctuations of normalized fuel vapor in the domain $(0.0 \mathrm{~mm}<x<1.8 \mathrm{~mm})$ and downstream region $(1.5 \mathrm{~mm}<x<1.8 \mathrm{~mm})$ of the oscillating case, the values for the two regions in steady case are set to unity.

Blue dotted line indicates crossflow oscillation for comparison of phase delay.

Figure 5 shows the fluctuations of the droplet Sauter mean diameter $\left(D_{32}\right)$ and arithmetic mean diameter $\left(D_{10}\right)$ in the whole computational domain in comparison with those values at the downstream region $(1.5 \mathrm{~mm}<x<1.8 \mathrm{~mm})$, which could be considered as the out-flow values, as well as the ones in a steady case of the mean crossflow velocity of $24.8 \mathrm{~m} / \mathrm{s}$. Even though there is a slight lag in phase, $D_{10}$ and $D_{32}$ of the oscillating case show periodic changes as the crossflow velocity changes, confirming that the atomization characteristics of the liquid jet change with crossflow velocity rapidly. $\mathrm{D}_{10}$ show smallest values when the crossflow velocity reaches the peak, and show peak values when the crossflow velocity is the smallest, which are in contrast to the $D_{32}$ values. During the most of the oscillating case, both $D_{32}$ and $D_{10}$ values in the whole computational domain and the out-flow ones are smaller than the steady case. The out-flow value of $D_{32}$ is smaller than the whole computational domain one, whereas the $D_{10}$ values show an inverse tendency. It could be considered as the result that the small droplets in the computational domain are totally consumed before flowing out.

Figure 6 shows the fluctuation of the ratio of fuel vapor of the oscillating case to the value of the steady case in the whole computational domain in comparison with the value at the 
downstream region $(1.5 \mathrm{~mm}<x<1.8 \mathrm{~mm})$. Both values in the oscillating case show periodic cosine-like curves. The small values could be found at the phase of $90^{\circ}$ and the peak values at $270^{\circ}$, which matches to the $D_{10}$ fluctuation very well. Compared to the value in domain, the out-flow (downstream region) one shows more violent change confirming that more fuel vapor are flown out of the computational domain due to the oscillating crossflow condition.

\section{Conclusions}

The characteristics of a liquid jet atomization under the oscillating crossflow were found to correspond to the air velocity rapidly so that the atomization characteristics showed continuous change as aerodynamic Weber number changes. The atomization process near the transition point was found to be extremely enhanced during the whole atomization process by investigating the atomized Eulerian components. The dispersed droplet profiles of the whole domain as well as the flow-out ones showed periodic changes as crossflow velocity changes. The evaporation effects were also found to be enhanced by comparing the $D_{10}$ (arithmetic mean diameter) and fuel vapor fluctuations.

\section{Acknowledgments}

The authors are grateful to Dr. K. Matsuura of Japan Aerospace Exploration Agency (JAXA) for many useful discussions. This work was partially supported by MEXT as "Program for Promoting Researches on the Supercomputer Fugaku" (Digital Twins of Real World's Clean Energy Systems with Integrated Utilization of Super-simulation and $\mathrm{Al}$ ) and by JSPS KAKENHI Grant Number $19 \mathrm{H} 02076$.

\section{References}

[1] Wen, J., Hu, Y., Nakanishi, A., Kurose, R., 2020, International Journal of Multiphase Flow, 103331.

[2] Sallam, K.A., Aalburg, C., Faeth, G.M., 2004, AIAA Journal, 42, pp. 2529-2540.

[3] Song, J., Cain, C.C., Lee, J., 2015, Journal of Engineering Gas Turbine Power Trans ASME, 137, 041502.

[4] Wu, P.K., Kirkendall, K.A., Fuller, R.P., Nejad, A.S., 1997, Journal of Propulsion Power, 13, pp. 64-73.

[5] Herrmann, M., 2011, Proceedings of Combustion Institute, 33, pp. 2079-2088.

[6] Li, X., Gao, H., Soteriou, M.C., 2017, Physics Fluids 29, 082103.

[7] Tachibana, S., Saito, K., Yamamoto, T., Makida, M., Kitano, T., Kurose, R., 2015, Combustion and Flame, 162, pp.2621-2637.

[8] Bunce, K., Lee, J.G., Santavicca, D.A., 2006, 41th AIAA Aerospace Sciences Meeting and Exhibit, AIAA2006-1225.

[9] Song, J., Ramasubramanian, C., Lee, J., 2014, Atomization and Sprays, 24, pp. 129-154. [10] Kurose, R., 2021, http://www.tse.me.kyoto-u.ac.jp/members/kurose/link e.php.

[11] Kitano, T., Nishio, J., Kurose, R., Komori, S., 2014, Fuel, 136, pp. 219-225.

[12] Kitano, T., Nishio, J., Kurose, R., Komori, S., 2014, Combustion and Flame, 170, pp. 6378.

[13] Albdadawi, A., Donoghue, D.B., Robinson, A.J., Murray, D.B., Delaure, Y.M.C., 2013, International Journal of Multiphase Flow, 53, pp. 11-28.

[14] Yokoi, K., 2013, Journal of Computational Physics, 232, pp. 252-271.

[15] Brackbill, J.U., Kothe, D.B., Zemach, C., 1992, Journal of Computational Physics, 100, pp. 335-354.

[16] Herrmann, M., 2010, Journal of Computational Physics, 229, pp. 745-759.

[17] Li, X., Soteriou, M.C., Arienti, M., Sussman, M.M., 2011, 49th AIAA Aerospace Sciences Meeting including the New Horizons Forum and Aerospace Exposition, AIAA2011-99. 